\title{
Lactobacillus rhamnosus GG Activation of Dendritic Cells and Neutrophils Depends on the Dose and Time of Exposure
}

\author{
Shirong Cai, ${ }^{1}$ Matheswaran Kandasamy, ${ }^{1}$ Juwita N. Rahmat, ${ }^{1}$ Sin Mun Tham, \\ Boon Huat Bay, ${ }^{2}$ Yuan Kun Lee, ${ }^{3}$ and Ratha Mahendran ${ }^{1}$ \\ ${ }^{1}$ Department of Surgery, Yong Loo Lin School of Medicine, National University of Singapore, Singapore 119228 \\ ${ }^{2}$ Department of Anatomy, Yong Loo Lin School of Medicine, National University of Singapore, Singapore 117594 \\ ${ }^{3}$ Department of Microbiology and Immunology, Yong Loo Lin School of Medicine, National University of Singapore, Singapore 117545 \\ Correspondence should be addressed to Ratha Mahendran; surrm@nus.edu.sg
}

Received 10 March 2016; Revised 9 May 2016; Accepted 19 May 2016

Academic Editor: Lenin Pavón

Copyright (C) 2016 Shirong Cai et al. This is an open access article distributed under the Creative Commons Attribution License, which permits unrestricted use, distribution, and reproduction in any medium, provided the original work is properly cited.

\begin{abstract}
This study evaluates the ability of Lactobacillus rhamnosus GG (LGG) to activate DC and neutrophils and modulate T cell activation and the impact of bacterial dose on these responses. Murine bone marrow derived DC or neutrophils were stimulated with LGG at ratios of $5: 1,10: 1$, and $100: 1$ (LGG: cells) and DC maturation (CD40, CD80, CD86, CD83, and MHC class II) and cytokine production (IL-10, TNF- $\alpha$, and IL-12p70) were examined after $2 \mathrm{~h}$ and $18 \mathrm{~h}$ coculture and compared to the ability of BCG (the present immunotherapeutic agent for bladder cancer) to stimulate these cells. A $2 \mathrm{~h}$ exposure to $100: 1$ (high dose) or an $18 \mathrm{~h}$ exposure to $5: 1$ or $10: 1$ (low dose), LGG : cells, induced the highest production of IL-12 and upregulation of CD40, CD80, CD86, and MHC II on DC. In DCs stimulated with LGG activated neutrophils IL-12 production decreased with increasing dose. LGG induced 10-fold greater IL-12 production than BCG. T cell IFN $\gamma$ and IL-2 production was significantly greater when stimulated with DC activated with low dose LGG. In conclusion, DC or DC activated with neutrophils exposed to low dose LGG induced greater Thl polarization in T cells and this could potentially exert stronger antitumor effects. Thus the dose of LGG used for immunotherapy could determine treatment efficacy.
\end{abstract}

\section{Introduction}

Lactobacillus rhamnosus GG (LGG) is a gram positive lactic acid bacterium that is part of the commensal microflora in humans. It is generally regarded as safe and has been used extensively in food products and health supplements. LGG has been reported to alleviate allergies and dermatitis $[1,2]$. Meta-analysis of probiotic supplementation during pregnancy and early infancy indicates a reduced risk ratio of developing eczema in early infancy [3]. A meta-analysis of LGG supplementation showed increased treatment responders in subjects with abdominal pain related gastrointestinal disorders and Irritable Bowel Syndrome [4]. Ohashi et al. also found that long-term consumption of Lactobacillus casei was associated with the reduced risk of bladder cancer [5]. LGG was also shown to possess antitumor effects in animal models of bladder cancer [6,7]. The antitumor effects were comparable to that induced by Mycobacterium bovis, bacillus Calmette-Guérin [7]. Intravesical instillations of LGG resulted in an influx of dendritic cells (DCs) and neutrophils [7]. Despite BCG's efficacy it is associated with significant side-effects and less toxic therapies are needed [8]; thus this study further evaluates the immunotherapeutic potential of LGG.

DCs are antigen presenting cells that play an important role in cancer immunotherapy by stimulating cytotoxic $\mathrm{T}$ lymphocytes (CTL) and polarizing $\mathrm{T}$ helper cells towards a Thl profile. DC maturation causes enhanced expression of surface costimulatory molecules and production of cytokines and chemokines. However, extensive stimulation of DC can result in DC exhaustion that is characterized by diminished production of IL-12 [9] which is necessary for CTL induction and interferon gamma (IFN $\gamma$ ) [10] production. DC exhaustion can be the result of prolonged exposure to a stimulus 
or exposure to a very high dose of a stimulus either of which scenarios are possible when analyzing the interaction of microbes with immune cells.

Miyazaki et al. [11] showed that, upon inflammation, neutrophils migrate from the site of infection to neighboring lymph nodes where they undergo apoptosis and are taken up by DCs, thus ensuring that neutrophil derived antigens are presented to T cells. Neutrophils are also able to directly transfer antigens to DCs as was demonstrated by Morel et al. [12], studying BCG infected neutrophils.

This work evaluates the impact of the dose of LGG and time of exposure on DC activation in the absence and presence of neutrophils and the consequent stimulation of $\mathrm{T}$ cells. The mouse orthotopic tumor model used to assess the intravesical instillation of LGG into the bladder followed the clinical protocol of BCG immunotherapy and was performed over a 2-hour time frame [7]. Thus, this was the minimum time of interaction that was analyzed and $18 \mathrm{~h}$ was chosen as the maximum time of interaction as, beyond this time frame, DC viability was greatly reduced after exposure to a high dose of LGG. The death induced by LGG on longer exposure may be a consequence of lactic acid production as observed with cancer cells exposed to LGG [13].

\section{Materials and Methods}

2.1. Bacterial Preparation. L. rhamnosus GG (National Collections of Industrial and Marine Bacterial Ltd., UK) was streaked onto deMan Rogosa Sharpe (MRS) agar (Difco Laboratories, USA) and incubated at $37^{\circ} \mathrm{C}$ in $5 \% \mathrm{CO}_{2}$ [14]. Single colonies were used to produce seed cultures $(9 \mathrm{~h})$ which were used to start $50 \mathrm{~mL}$ cultures. Bacteria were harvested at the late log phase by centrifugation $(1699 \times \mathrm{g}$ for 10 minutes at room temperature) and washed twice with sterile saline $(0.85 \% \mathrm{NaCl})$. The colony forming units (CFU) were determined by plating serial dilutions of the bacterial samples on MRS agar plates which were incubated at $37^{\circ} \mathrm{C}$ in $5 \% \mathrm{CO}_{2}$. BCG Connaught was prepared in the lab as previously described [15].

2.2. Preparation of Bone Marrow Derived Neutrophils, DC, and $T$ Cells. Protocols were approved by the Institutional Animal Care and Use Committee (IACUC) at the National University of Singapore. Bone marrow derived neutrophils and DC were generated as previously described [16]. In brief, neutrophils were derived by positive selection with anti-Ly6G microbead kit (Miltenyi Biotec, Germany) and were at least 95\% positive for Ly6G, by flow cytometry. DCs were obtained from the bone marrow derived cells after 9 days of culture (with fresh media replacement every other day) in RPMI 1640 supplemented with $10 \%$ heat-inactivated FCS, $50 \mu \mathrm{M}$ 2-mercaptoethanol, $1 \%$ penicillin, streptomycin, glutamine, MEM (minimum essential medium), and $0.1 \%$ sodium pyruvate with $40 \mathrm{ng} / \mathrm{mL}$ of GM-CSF (BD Bioscience, USA). The DCs were at least $95 \%$ positive for CD11c, by flow cytometry. The media used for both neutrophil and DC experiments were DMEM supplemented with $10 \%$ fetal bovine serum (Hyclone, USA), $2 \mathrm{mM}$ L-glutamine (Gibco,
Japan), $50 \mu \mathrm{g} / \mathrm{mL}$ penicillin G (Sigma Aldrich, USA), and $50 \mu \mathrm{M}$ of 2 -mercaptoethanol (Merck, Germany) with the addition of $20 \mathrm{ng} / \mathrm{mL}$ of GM-CSF for the culture of dendritic cells.

$\mathrm{T}$ lymphocytes were isolated from spleens of naive C57BL/6 mice and enriched with the EasySepTM T cell isolation kit (STEMCELL Technologies, Vancouver, Canada). The desired fraction was about $95-98 \%$ CD3 positive.

2.3. Neutrophil, Dendritic Cell LGG Coculture, and Blocking of IL-10 and COX-2. The LGG to cell ratios of $5: 1$ and 10:1 were defined as exposure to low dose LGG, while exposure to a ratio of $100: 1$ was defined as exposure to high dose LGG. Neutrophils $\left(5 \times 10^{5}\right)$ and DCs $(2.5 \times$ $10^{5}$ ) were cocultured with LGG at bacteria to mammalian cell ratios of $5: 1,10: 1$, and $100: 1$ for $2 \mathrm{~h}$ in 24 -well plates, before $200 \mu \mathrm{g} / \mathrm{mL}$ of gentamicin was added for $2 \mathrm{~h}$ at $37^{\circ} \mathrm{C}$ to kill extracellular LGG. Cells were washed thrice with PBS to remove extracellular bacteria and then neutrophils were incubated with DCs $\left(2.5 \times 10^{5}\right)$ for $18 \mathrm{~h}$ and DCs were incubated in fresh media for $18 \mathrm{~h}$. Untreated neutrophils and DCs were evaluated as controls. All controls were given the same treatment as above. For $2.5 \times 10^{5} \mathrm{DCs}$ the bacteria CFU that corresponded to $5: 1,10: 1$, and $100: 1$ were $1.25 \times 10^{6}$, $2.5 \times 10^{6}$, and $25 \times 10^{6} \mathrm{CFU}$. The neutrophils $\left(5 \times 10^{5}\right.$ cells $)$ were treated with $2.5 \times 10^{6}, 5 \times 10^{6}$, and $50 \times 10^{6} \mathrm{CFU}$ of LGG that corresponded to $5: 1,10: 1$, and $100: 1$, LGG to cells. For the DC $18 \mathrm{~h}$ experiment the DCs were exposed to LGG for $18 \mathrm{~h}$. Similarly cells were treated with BCG at a 5:1 ratio. The supernatants were assayed for TNF- $\alpha$, IL-12p70, and IL-10 (eBioscience, San Diego, USA) and prostaglandin E2 (PGE2) (Cayman Chemical, USA) by ELISA using a GENios Pro $^{\text {TM }}$ microplate reader (Tecan, Switzerland). The cells were harvested in PBA (PBS with $1 \%$ bovine serum albumin and $0.01 \%$ sodium azide) for flow analysis of surface markers.

IL-10 and COX-2 were inhibited by pretreatment with $400 \mathrm{ng} / \mathrm{mL}$ of anti-IL-10 antibody (Biolegend, San Diego, USA) and $10 \mu \mathrm{M}$ of NS398 (Sapphire Bioscience, Australia), respectively, for $30 \mathrm{mins}$ at $37^{\circ} \mathrm{C}$ prior to addition of bacteria and then further incubated for another 18 hours. The respective isotype and solvent controls were included for comparison. The efficacy of the blocking was confirmed by ELISA.

2.4. Flow Cytometry and Antibodies. Fixed DCs were double stained with anti-CD11c antibody and antibodies of the following surface markers: CD40, CD80, CD83, CD86, and MHC II (Biolegend) or the respective isotype controls in PBA (1x PBS with $1 \%$ BSA, $0.05 \%$ sodium azide) for 20 mins at $4^{\circ} \mathrm{C}$. After that the cells were washed once and resuspended with PBA before they were analyzed with BD FACS Canto using FACS Diva software (Becton Dickinson, USA).

2.5. DC-T Cell Coculture. DCs or T cells were resuspended in LDA medium (NCTC 109 and RPMI 1640 [1:1]), supplemented with $10 \%$ heat-inactivated FCS, $10 \mathrm{mM}$ L-glutamine, $1 \mathrm{mM}$ oxaloacetic acid, $0.2 \mathrm{U}$ of bovine insulin per $\mathrm{mL}$, and $50 \mu \mathrm{M} 2$-mercaptoethanol. Naive T cells $\left(1.0 \times 10^{7}\right.$ cells $\left./ \mathrm{mL}\right)$ 
TABLE 1: Surface marker expression on DC after direct and indirect exposure via neutrophils to BCG and LGG.

\begin{tabular}{|c|c|c|c|c|c|c|}
\hline \multirow{2}{*}{ Marker } & \multirow{2}{*}{$\mathrm{DC}$} & \multicolumn{5}{|c|}{ Percentage of CD11c ${ }^{+}$cells } \\
\hline & & Control & $5: 1$ (BCG) & $5: 1(\mathrm{LGG})$ & $10: 1$ (LGG) & $100: 1$ (LGG) \\
\hline \multirow{3}{*}{ CD40 } & $2 \mathrm{~h}$ & $6.0 \pm 3.3$ & $22.5 \pm 3.3$ & $32.1 \pm 4.9$ & $32.7 \pm 0.3$ & $50.7 \pm 2.6^{\mathrm{b}}$ \\
\hline & $18 \mathrm{~h}$ & $2.2 \pm 0.5$ & $19.3 \pm 5.4$ & $20.5 \pm 6.9$ & $19.0 \pm 6.8$ & $7.0 \pm 0.8^{\mathrm{b}}$ \\
\hline & +neutrophils $(2 \mathrm{~h})$ & $2.2 \pm 0.4$ & $27.8 \pm 3.5$ & $22.9 \pm 5.2$ & $25.4 \pm 8.7$ & $10.4 \pm 2.0^{\mathrm{b}}$ \\
\hline \multirow{3}{*}{ CD80 } & $2 \mathrm{~h}$ & $9.9 \pm 1.4$ & $39.3 \pm 10.4$ & $18.2 \pm 0.8$ & $18.1 \pm 3.3$ & $34.0 \pm 4.2^{\mathrm{b}}$ \\
\hline & $18 \mathrm{~h}$ & $9.6 \pm 1.6$ & $30.7 \pm 7.5$ & $17.9 \pm 0.5$ & $19.7 \pm 2.5$ & $19.6 \pm 0.8$ \\
\hline & +neutrophils ( $2 \mathrm{~h})$ & $6.1 \pm 1.6$ & $33.8 \pm 8.9$ & $21.2 \pm 5.0$ & $16.6 \pm 7.6$ & $16.1 \pm 7.8$ \\
\hline \multirow{3}{*}{ CD83 } & $2 \mathrm{~h}$ & $6.5 \pm 0.2$ & $29.6 \pm 5.7$ & $38.4 \pm 3.7$ & $32.7 \pm 1.3$ & $36.2 \pm 4.7$ \\
\hline & $18 \mathrm{~h}$ & $5.1 \pm 1.5$ & $27.5 \pm 10.5$ & $8.4 \pm 1.9$ & $4.8 \pm 0.8$ & $6.5 \pm 1.4$ \\
\hline & +neutrophils ( $2 \mathrm{~h})$ & $3.6 \pm 0.2$ & $31.2 \pm 4.8$ & $9.8 \pm 1.3$ & $8.0 \pm 0.8$ & $11.1 \pm 2.8$ \\
\hline \multirow{3}{*}{ CD86 } & $2 \mathrm{~h}$ & $17.0 \pm 0.3$ & $72.0 \pm 5.4$ & $37.1 \pm 8.1$ & $41.6 \pm 1.4$ & $58.5 \pm 6.1^{\mathrm{b}}$ \\
\hline & $18 \mathrm{~h}$ & $14.8 \pm 1.9$ & $71.0 \pm 7.9$ & $57.6 \pm 0.1$ & $56.5 \pm 1.3$ & $29.7 \pm 6.0^{\mathrm{b}}$ \\
\hline & +neutrophils ( $2 \mathrm{~h})$ & $13.7 \pm 0.2$ & $68.0 \pm 4.8$ & $68.3 \pm 6.4$ & $76.5 \pm 4.4$ & $54.9 \pm 3.8^{\mathrm{b}}$ \\
\hline \multirow{3}{*}{$\begin{array}{l}\text { MHC } \\
\text { class II }\end{array}$} & $2 \mathrm{~h} \mathrm{LGG}$ & $31.7 \pm 1.8$ & $61.9 \pm 2.5$ & $69.9 \pm 8.8$ & $70.7 \pm 12.8$ & $77.8 \pm 11.1$ \\
\hline & $18 \mathrm{~h} \mathrm{LGG}$ & $32.6 \pm 2.7$ & $59.2 \pm 6.1$ & $58.4 \pm 12.5$ & $52.9 \pm 7.2$ & $57.4 \pm 11.6$ \\
\hline & +neutrophils (2 h) & $34.7 \pm 10.1$ & $55.3 \pm 10.2$ & $58.5 \pm 12.9$ & $52.1 \pm 6.3$ & $64.0 \pm 9.4$ \\
\hline
\end{tabular}

${ }^{\mathrm{b}} p<0.05$ compared to groups treated with 5:1 and 10:1 LGG, respectively. Data are presented as the mean \pm SEM.

were cultured with untreated DCs $\left(1.0 \times 10^{5}\right.$ cells $\left./ \mathrm{mL}\right)$ or DCs stimulated with lactobacilli for $2 \mathrm{~h}$ and $18 \mathrm{~h}$ (treated as described above), in $200 \mu \mathrm{L}$ of LDA medium in 96-well Ubottom plates at $37^{\circ} \mathrm{C}$ under $5 \% \mathrm{CO}_{2}$ for 5 days. After 5 days, supernatants were harvested and analyzed for IFN gamma or IL-2.

2.6. Statistical Analysis. One-way ANOVA with post hoc Bonferroni test was used to analyze all the data except for the comparison of cytokine profile of the treatment groups with anti-IL-10 antibody or NS398 and their respective controls, which were analyzed with Student's $t$-test. A significant difference was taken to exist when the $p$ value was $<0.05$.

\section{Results}

3.1. LGG Dose, Exposure Time, and Neutrophils Modulate DC and Neutrophil Maturation and Viability. A short exposure $(2 \mathrm{~h})$ to low dose LGG (LGG to cell ratios of $10: 1$ and $5: 1)$ reduced DC viability slightly $(91.7 \pm 2.0 \%$ and $94.7 \pm$ $1.7 \%$, resp.) and there was little loss in viability even at $18 \mathrm{~h}$. Exposure to activated neutrophils had a similar effect. However at a prolonged exposure of $18 \mathrm{~h}$ to high dose LGG (100:1 LGG to DC ratio), there was reduced DC viability $(63.7 \pm 1.8 \%)$.

About $50 \%$ of neutrophils were dead (apoptotic and necrotic death) at $18 \mathrm{~h}$ after the initial $2 \mathrm{~h}$ exposure to LGG regardless of dose. But in contrast there was increased LGG internalized with exposure to increased LGG dose. At a 5:1 ratio of LGG: neutrophils there were $228 \pm 51 \mathrm{CFU}$ internalized $/ 5 \times 10^{5}$ neutrophils and this almost doubled after exposure to $10: 1$ and more than doubled again after exposure to $100: 1$ LGG to neutrophils. The LGG in the neutrophils were still viable at $18 \mathrm{~h}$ after internalization.

Activation markers on naïve DC were examined and after exposure to LGG there was a significant increase in all markers with respect to naive DC, Table 1 . As a further control DCs were also exposed to BCG, Table 1. After high dose LGG exposure for $2 \mathrm{~h}$, there was significantly $(p<0.05)$ higher expression of CD80, CD86, and CD40 compared to low dose LGG. But at $18 \mathrm{~h}$ coincubation with LGG, expression of CD86 and CD40 was significantly reduced $(p<0.05)$ after exposure to high dose LGG compared to low dose LGG. DCs cocultured with neutrophils, activated with low doses of LGG for $2 \mathrm{~h}$, showed higher expression of CD86 and reduced CD83 compared to DCs exposed directly to low dose LGG for $2 \mathrm{~h}$. DC exposed to BCG at a dose of $5: 1$, for $2 \mathrm{~h}$ or $18 \mathrm{~h}$, or stimulated by neutrophils activated with BCG did not show a difference in surface marker expression. In contrast LGG at the same dose showed changes in the expression of CD83 and CD86.

Neutrophils cultured with LGG showed decreased MHC class I expression, no increase in MHC class II expression, and an increase in CD11b expression when placed directly in contact with LGG. CD11b is an activation marker for neutrophils and has been shown to activate DC maturation via interaction with DC-SIGN [17].

MHC class II mean fluorescence index (MFI) showed a doubling on exposure to low dose LGG or activated neutrophils, Table 2. The MFI for MHC class II, CD40, and CD80 was decreased after exposure to DC activated for $18 \mathrm{~h}$, with either high dose LGG or neutrophils activated with high dose LGG $(p<0.05)$. But the reverse was true for CD83 when DCs were exposed to high dose LGG for $18 \mathrm{~h}$ 
TABLE 2: LGG dose and time of exposure modulated the MFI of DC surface markers.

\begin{tabular}{|c|c|c|c|c|c|}
\hline Marker & Group & Control & $5: 1$ & $10: 1$ & $100: 1$ \\
\hline \multirow{3}{*}{$\begin{array}{l}\text { MHC } \\
\text { class II }\end{array}$} & $\mathrm{DC} 2 \mathrm{~h}$ & $1191 \pm 10$ & $2522 \pm 2^{\mathrm{a}}$ & $2545 \pm 94^{\mathrm{a}}$ & $2978 \pm 245^{\mathrm{a}}$ \\
\hline & $\mathrm{DC} 18 \mathrm{~h}$ & $1303 \pm 37$ & $2066 \pm 154^{\mathrm{a}}$ & $2124 \pm 29^{\mathrm{a}}$ & $1530 \pm 3^{\mathrm{bc}}$ \\
\hline & DC + neutrophils & $1191 \pm 28$ & $2010 \pm 107^{\mathrm{a}}$ & $2352 \pm 78^{\mathrm{a}}$ & $1946 \pm 80^{\mathrm{ac}}$ \\
\hline \multirow{3}{*}{$\mathrm{CD} 40$} & $\mathrm{DC} 2 \mathrm{~h}$ & $539 \pm 1$ & $629 \pm 1^{\mathrm{a}}$ & $654 \pm 1^{\mathrm{a}}$ & $684 \pm 6^{\mathrm{abc}}$ \\
\hline & $\mathrm{DC} 18 \mathrm{~h}$ & $489 \pm 42$ & $735 \pm 27^{\mathrm{a}}$ & $721 \pm 1^{\mathrm{a}}$ & $370 \pm 8^{\mathrm{bc}}$ \\
\hline & DC + neutrophils & $437 \pm 61$ & $576 \pm 15$ & $642 \pm 18^{\mathrm{a}}$ & $390 \pm 21^{c}$ \\
\hline \multirow{3}{*}{ CD83 } & $\mathrm{DC} 2 \mathrm{~h}$ & $465 \pm 20$ & $417 \pm 11$ & $554 \pm 79$ & $452 \pm 22$ \\
\hline & $\mathrm{DC} 18 \mathrm{~h}$ & $668 \pm 20$ & $506 \pm 33^{\mathrm{a}}$ & $681 \pm 7^{b}$ & $702 \pm 12^{\mathrm{b}}$ \\
\hline & DC + neutrophils & $704 \pm 34$ & $609 \pm 61^{a}$ & $441 \pm 54^{\mathrm{a}}$ & $351 \pm 3^{\mathrm{ab}}$ \\
\hline \multirow{3}{*}{ CD80 } & $\mathrm{DC} 2 \mathrm{~h}$ & $515 \pm 4$ & $537 \pm 5$ & $516 \pm 14$ & $568 \pm 15^{\mathrm{ac}}$ \\
\hline & $\mathrm{DC} 18 \mathrm{~h}$ & $513 \pm 3$ & $544 \pm 12$ & $535 \pm 10$ & $478 \pm 28^{\mathrm{b}}$ \\
\hline & DC + neutrophils & $476 \pm 6$ & $613 \pm 16^{\mathrm{a}}$ & $553 \pm 10^{\mathrm{a}}$ & $478 \pm 21^{\mathrm{bc}}$ \\
\hline
\end{tabular}

${ }^{\mathrm{a}} p<0.05$ compared to control with no bacteria.

${ }^{\mathrm{b}, \mathrm{c}} p<0.05$ compared to groups treated with $5: 1$ and $10: 1 \mathrm{LGG}$, respectively. Data are presented as the mean \pm SEM.

$(p<0.05)$, Table 2 . The MFI of CD86 did not vary with treatment conditions.

3.2. LGG Dose, Exposure Time, and Neutrophils Modulate DC Cytokine Production. More IL-10 was produced after exposure to high dose LGG (Figure 1(a)) for $2 \mathrm{~h}$ and $18 \mathrm{~h}$ and via neutrophil mediated activation. TNF- $\alpha$ production was higher in DC exposed to high dose LGG (Figure 1(a)) for $2 \mathrm{~h}$, but at $18 \mathrm{~h}, \mathrm{DC}$ exposed to low dose LGG produced more TNF- $\alpha$. Both indirect (via neutrophils) and direct DC activation for $18 \mathrm{~h}$ resulted in more IL-12p70 production after low dose LGG exposure (Figure 1(a)). With a short $2 \mathrm{~h}$ exposure to LGG, IL-12p70 production was independent of the bacterial dose.

Neutrophils stimulated with LGG produced IL-12p70 and TNF- $\alpha$ and very little IL-10. LGG activated neutrophils ( $2 \mathrm{~h}$ ) induced more IL-10, TNF- $\alpha$, and IL-12p70 production in DC compared to DC exposed directly to low dose LGG for $2 \mathrm{~h}$. At high dose LGG there was no significant difference in IL-12p70 and TNF- $\alpha$ production, whether the DC was stimulated directly with LGG or with activated neutrophils.

In contrast, when DC and neutrophils were exposed to BCG at a 5:1 ratio [12], there was comparable production of IL-10 from all groups except DC exposed to BCG for $18 \mathrm{~h}$. The amount of IL-12p70 produced after BCG stimulation was at least 10 -fold lower than that produced by LGG. The TNF- $\alpha$ response was comparable to LGG (Figure 1(a)).

3.3. IL-12p70 Production after Exposure to LGG Stimulated Neutrophils Is Dependent on IL-10. Since, at high dose LGG, IL-10 production is significantly higher in DCs, as well as DC treated with activated neutrophils, we determined if the dose dependent effects on IL-12 production were due to IL-10 levels. $\mathrm{PGE}_{2}$ is known to modulate IL-10 expression; induce indoleamine 2,3-dioxygenase (a potent suppressor of DC function); and modulate chemokine production and DC maturation and IL-12p70 production [18-20]. Therefore
$\mathrm{PGE}_{2}$ and IL-10 production/function were inhibited individually and the impact on IL-12p70 production was monitored, Figure 1(b). There was a significant increase in $\mathrm{PGE}_{2}$ levels on DC stimulation with high rather than low dose LGG. At the concentration of NS398 that completely blocked the production of $\mathrm{PGE}_{2}$ (Figure 1(b)) there was no significant effect on either IL-10 or IL-12p70 levels, Figure 1(b). Blocking IL-10 with a neutralizing antibody caused a sharp increase in IL-12p70 production, Figure 1(b). This corresponded to 2.1- and 4.4-fold increases, respectively, in DC stimulated with neutrophils activated with low and high dose LGG. Expression of surface markers on dendritic cells was not significantly affected by the presence of either the anti-IL-10 antibody or NS398 (data not shown).

3.4. T Cell Activation Is Dependent on LGG to DC Ratios and Time of Exposure. Neutrophils stimulated with LGG did not induce IFN $\gamma$ production by T cells (Figure 2(a)) but induced IL-2 production (Figure 2(b)). DCs stimulated with LGG activated neutrophils induced a significant increase $(p<$ 0.05 ) in IFN $\gamma$ production (Figure 2(a)) and a slight increase in IL-2 production by T cells (Figure 2(b)) similar to DC directly activated with low dose LGG. The DC-neutrophil$\mathrm{T}$ cell triple cell culture by itself induced IL- 2 production. A dose dependent effect was clearly seen with IFN $\gamma$ production $(p<0.05)$ (Figure 2(a)) which was consistent with the decrease in IL-12p70 production that was observed earlier. Direct or indirect DC activation with low dose LGG for $2 \mathrm{~h}$ induced more IFN $\gamma$ and IL-2 production by T cells $(p<0.05$ for IFN $\gamma$ ) compared to high dose LGG, Figures 2(a) and 2(b). At $18 \mathrm{~h}$ the differential effect of the dose was lost for IFN $\gamma$.

\section{Discussion}

On activation by LGG, there was an increase in the percentage of DC expressing CD40, CD80, and CD86 with increasing dose. But only CD40 had a significant increase in MFI 

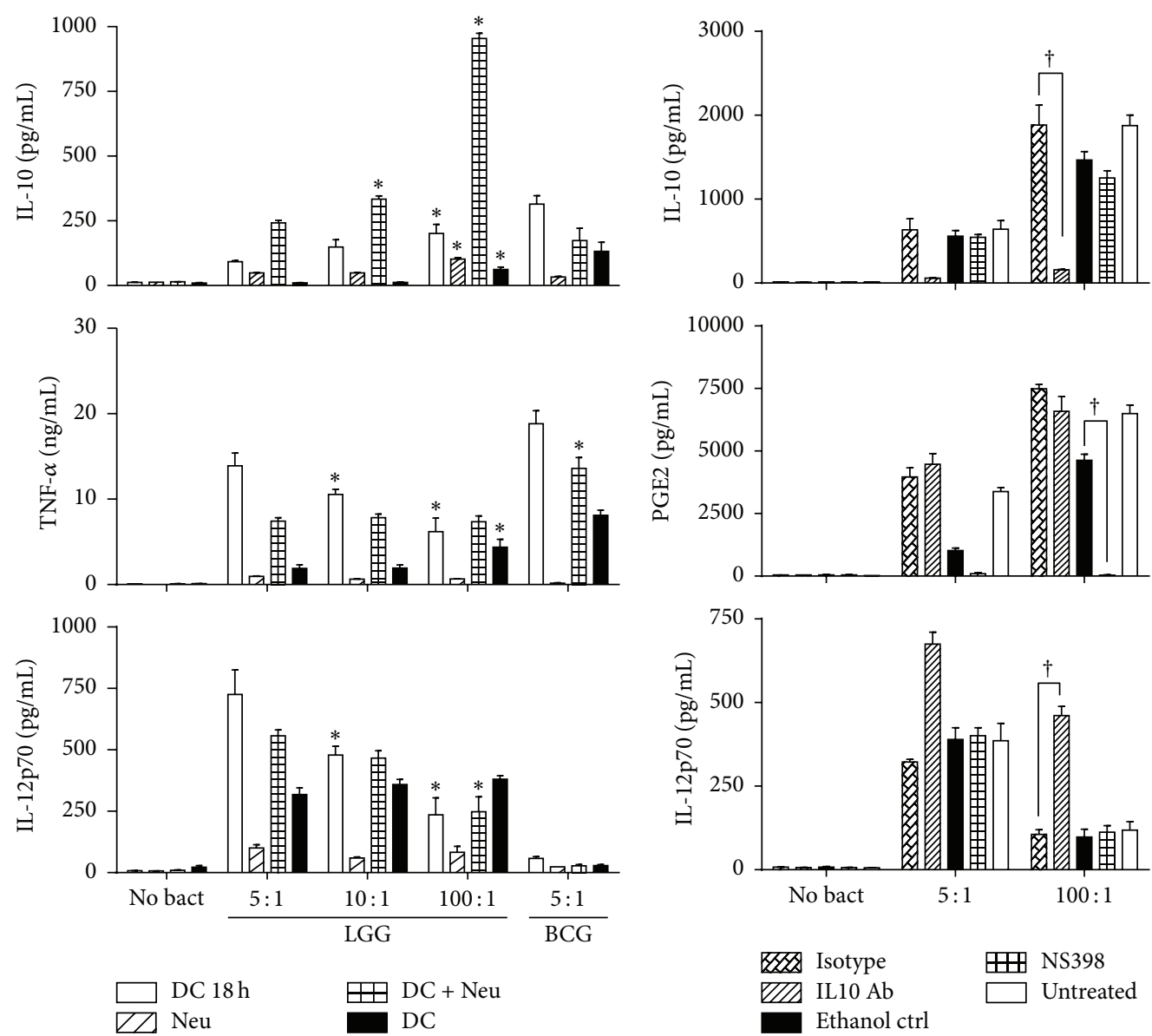

(a)

(b)

FIGURE 1: LGG and BCG induced dose and time dependent effects on DC cytokine production by direct or indirect stimulation via neutrophils. LGG was assessed at $5: 1,10: 1$, and $100: 1$ ratios and BCG at $5: 1$ ratios. (a) Production of IL-10, TNF- $\alpha$, and IL-12p70 after 18 h of continuous coculture of DCs with LGG/BCG (white bar), $2 \mathrm{~h}$ of exposure of DCs (black bar) or neutrophil (striped bar) to LGG/BCG, followed by $18 \mathrm{~h}$ of bacteria free incubation and $18 \mathrm{~h}$ of DC coculture with neutrophils pretreated with LGG/BGC for $2 \mathrm{~h}$ (crisscross bar). “*” indicates a significant difference $(p<0.05)$ compared to low dose $(5: 1)$. For BCG “*” indicates a significant difference from neutrophils and for TNF- $\alpha$ significance with respect to DC. Data are presented as the mean \pm SEM. (b) Neutrophils were prestimulated with low $(5: 1)$ and high (100:1) dose of LGG for $2 \mathrm{~h}$ before they were cocultured with DC for $18 \mathrm{~h}$ in the presence and absence of a COX-2 inhibitor, NS398 (crisscross bar); COX-2 inhibitor solvent control (black bar); IL-10 neutralizing antibody (IL-10 Ab) (striped bar); and the isotype control for the antibody (double striped bar). The impact on $\mathrm{PGE}_{2}$, IL-10, and IL-12p70 secretion is shown. " $\dagger$ " indicates a significant difference from the respective controls $(p<0.05)$. Data are presented as the mean \pm SEM.

$(p<0.05)$, indicating increased protein expression. CD86 and CD80 interact with CD28 on T cells while CD40 binds to the CD40 Ligand on T cells to induce T cell activation. On prolonged exposure $(18 \mathrm{~h})$ to high dose LGG, the percentage of cells expressing CD86 and CD40 and the MFI of CD40, CD86, and CD80 were reduced. Thus, these DCs may not be as able to activate $\mathrm{T}$ cells as efficiently; that is, there is a point beyond which LGG dose can be inhibitory to DC activation. A similar effect was observed with L. casei [21] which also has antitumor effects [22] and other commensal lactobacilli such as L. gasseri, L. johnsonii, and L. reuteri [23].

Different Lactobacillus species induce variable levels of IL-10, IL-12, and TNF- $\alpha$ via Toll-like receptor (TLR) dependent activation of DC [24]. Indirect DC stimulation via LGG activated neutrophils showed no difference in TNF$\alpha$ production with increasing dose. But primary interaction between DC and LGG showed dose dependent effects. This could be due to TLR engagement and phagocytosis [25]. LGG is known to adhere to epithelial cells with greater ability than other Lactobacillus species. Such binding to DC may also increase the cellular signals triggered by direct interaction with DC. Tytgat et al. found that LGG pili S SpaCBA could interact with DC-SIGN and that blocking this interaction reduced DC cytokine production [26]. DCSIGN also modulates TLR activation and it is possible that LGG pili interaction with DC-SIGN could have modulated TLR activated cytokine production. An $18 \mathrm{~h}$ exposure to low dose LGG produced more TNF- $\alpha$ and IL-12 than exposure to high dose LGG. It is likely that prolonged exposure led to increasing phagocytosis of LGG with time which resulted in triggering the downregulation of TNF- $\alpha$ and IL-12 production. IL-12 production is TLR2 independent [25] but 


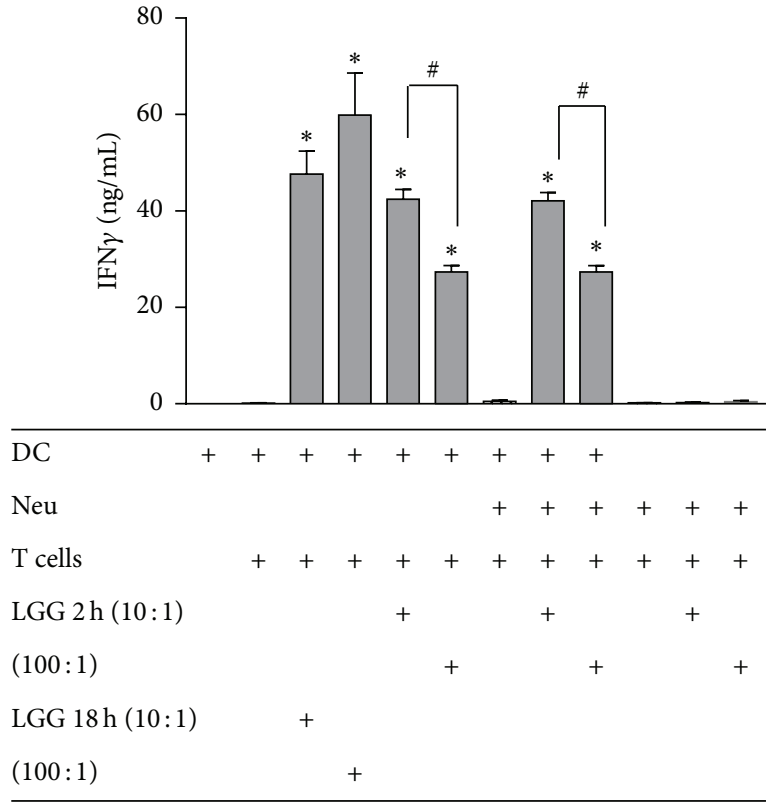

(a)

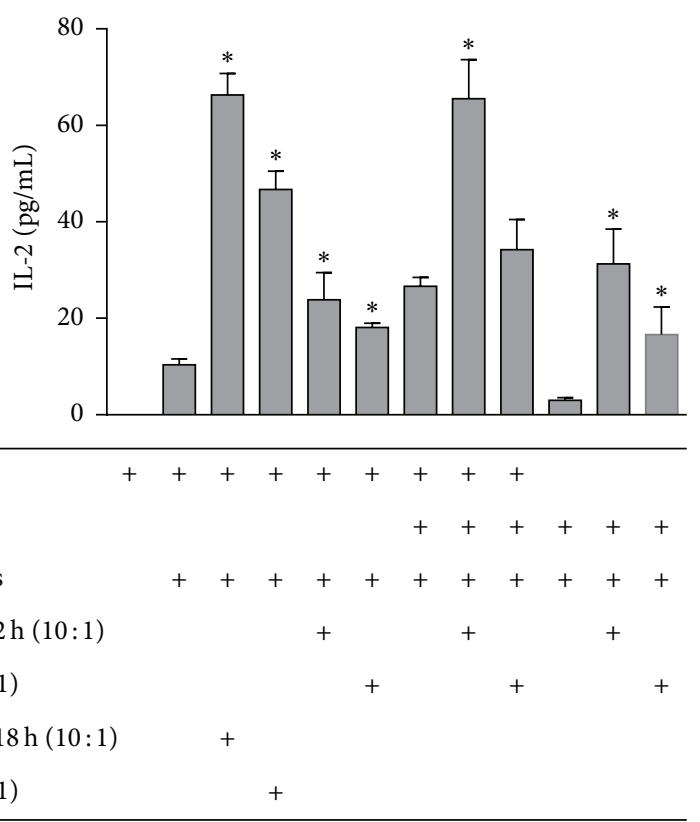

(b)

Figure 2: T cell activation is dependent on the dose of LGG used to stimulate DC or neutrophils. IFN $\gamma$ (a) and IL-2 (b) production by T cells after 5 days of coculture with DC, neutrophil, or DC-neutrophil (stimulated with low or high dose of LGG for $2 \mathrm{~h}$ and then DC for $18 \mathrm{~h}$ ). Data are presented as the mean \pm SEM. “*” indicates a significant difference compared to their respective no LGG controls $(p<0.05)$. “\#” indicates significant difference between high and low dose of LGG treatment $(p<0.05)$.

TLR9 [27], MyD88, and ROS [28] dependent. Phagocytosis of LGG is important, as is the presence of undigested bacteria [29]. However, with a short exposure to LGG there was a dose dependent effect only on TNF- $\alpha$ production which might reflect TLR2 engagement [25].

As neutrophils are generally the first to encounter microbes and move to the lymph nodes to educate DC [30], we evaluated the dose effect of LGG on the ability of neutrophils to activate DC. Stimulating DC with LGG treated neutrophils exposed to low dose LGG induced higher CD86 than direct stimulation of DCs with LGG. Neutrophils cultured with high dose LGG induced a decrease in the MFI of CD40, CD80, and CD83 $(p<0.05)$ on DCs. Though the number of neutrophils undergoing apoptosis was similar at all doses of LGG the number of internalized LGG increased with dose of LGG. The latter may have resulted in increased LGG or LGG components being transferred to DC [11, 12] causing strong stimulation of DC and consequently DC exhaustion. In line with this hypothesis, IL-12p70 and TNF- $\alpha$ production were much higher when the DCs were cultured with neutrophils activated with low dose LGG. DCs are known to internalize apoptotic cells [31] which like necrotic cells are able to stimulate DC [32]. Phagocytosis of apoptotic cells induces anti-inflammatory signals [33] such as the high levels of IL-10 which was found in this study.

IFN $\gamma$ production was higher in T cells cocultured with DC and neutrophils treated with low dose LGG for $2 \mathrm{~h}$ rather than high dose LGG. Similarly, when DCs were treated with LGG at 200:1 bacteria to cell ratio, phenotypic maturation and cytokine production but not Thl polarization were observed [21]. Instead, the $\mathrm{CD} 4^{+}$cells were converted to hyporesponsive $\mathrm{T}$ cells that secrete low IFN $\gamma$. Thus, for optimal T cell activation, low dose LGG is overall the better therapeutic option.

Prolonged stimulation of DCs (for $24 \mathrm{~h}$ or longer) can result in the loss of the ability to produce cytokines like IL-12, which is termed DC exhaustion $[9,34]$. These "exhausted" DCs tend to induce Th2 cell differentiation. Langenkamp et al. reported that the optimal temporal window to induce DC maturation in order to have sustained IL-12 production for cancer immunotherapy is narrow, with a time frame of 10-18 h [9], but our results indicate that a $2 \mathrm{~h}$ exposure is sufficient for DC maturation. Further LGG was much better at inducing IL-12p70 production than BCG, the current standard therapy for bladder cancer.

IL-10 is widely reported to downregulate DC maturation $[35,36]$ and its ability to activate T cells [37] as well as induce DC apoptosis [38]. $\mathrm{PGE}_{2}$, a potent inducer of IL-10 [20], was also found to be produced in greater amounts when DCs were stimulated with neutrophils treated with high dose LGG. Neutralization of IL-10 substantially increased the IL12 p70 production. However, it was still lower than the levels produced by DC coculture with neutrophil stimulated with low dose LGG, suggesting that there are other inhibitory factors aside from IL-10.

\section{Conclusion}

Low dose LGG stimulates DC to induce greater Th1 polarization in T cells compared to high dose LGG. Thus, low dose 
LGG would potentially be able to exert stronger antitumor effects. In mice LGG $\left(1 \times 10^{8} \mathrm{CFU} / 100 \mu \mathrm{L}\right)$ was effective at reducing tumor growth with comparable efficacy to BCG Connaught $\left(1 \times 10^{7} \mathrm{CFU} / \mathrm{mL}\right)$ [7]. The former is roughly in the range of a $100: 1$, LGG to cells for $2 \mathrm{~h}$. Thus future analysis should consider the effect of a 10 -fold lower dose of LGG as an immunotherapeutic agent. The dose response is an important consideration if LGG is to be used for human bladder cancer therapy.

\section{Abbreviations}

LGG: Lactobacillus rhamnosus GG

BCG: Bacillus Calmette-Guérin

DCs: Dendritic cells.

\section{Competing Interests}

None of the authors has competing interests.

\section{Authors' Contributions}

Shirong Cai and Matheswaran Kandasamy performed the DC and neutrophil studies with LGG and Juwita N. Rahmat performed the studies with BCG and DC and Sin Mun Tham performed the $\mathrm{PGE}_{2}$ analysis of LGG and DC. Boon Huat Bay, Yuan Kun Lee, and Ratha Mahendran conceived the experimental design. Shirong Cai, Juwita N. Rahmat, and Ratha Mahendran wrote the paper and the others reviewed it.

\section{Acknowledgments}

This work was funded by the Academic Research Fund, National University of Singapore, Yong Loo Lin School of Medicine, and a grant from the Biomedical Research Council Singapore (BMRC/04/1/21/19/311).

\section{References}

[1] T. Pessi, Y. Sütas, M. Hurme, and E. Isolauri, "Interleukin10 generation in atopic children following oral lactobacillus rhamnosus GG," Clinical and Experimental Allergy, vol. 30, no. 12, pp. 1804-1808, 2000.

[2] J. Sawada, H. Morita, A. Tanaka, S. Salminen, F. He, and H. Matsuda, "Ingestion of heat-treated Lactobacillus rhamnosus GG prevents development of atopic dermatitis in NC/Nga mice," Clinical and Experimental Allergy, vol. 37, no. 2, pp. 296303, 2007.

[3] G. Zuccotti, F. Meneghin, A. Aceti et al., "Probiotics for prevention of atopic diseases in infants: systematic review and meta-analysis," Allergy, vol. 70, no. 11, pp. 1356-1371, 2015.

[4] A. Horvath, P. Dziechciarz, and H. Szajewska, "Meta-analysis: Lactobacillus rhamnosus GG for abdominal pain-related functional gastrointestinal disorders in childhood," Alimentary Pharmacology \& Therapeutics, vol. 33, no. 12, pp. 1302-1310, 2011.

[5] Y. Ohashi, S. Nakai, T. Tsukamoto et al., "Habitual intake of lactic acid bacteria and risk reduction of bladder cancer," Urologia Internationalis, vol. 68, no. 4, pp. 273-280, 2002.
[6] B.-K. Lim, R. Mahendran, Y.-K. Lee, and B.-H. Bay, "Chemopreventive effect of Latobacillus rhamnosus on growth of a subcutaneously implanted bladder cancer cell line in the mouse," Japanese Journal of Cancer Research, vol. 93, no. 1, pp. 36-41, 2002.

[7] S. W. Seow, S. Cai, J. N. Rahmat et al., "Lactobacillus rhamnosus GG induces tumor regression in mice bearing orthotopic bladder tumors," Cancer Science, vol. 101, no. 3, pp. 751-758, 2010.

[8] Z. S. Yuksel, E. Buber, T. Kocagoz, A. Alp, Z. Saribas, and N. L. Acan, "Mycobacterial strains that stimulate the immune system most efficiently as candidates for the treatment of bladder cancer," Journal of Molecular Microbiology and Biotechnology, vol. 20, no. 1, pp. 24-28, 2011.

[9] A. Langenkamp, M. Messi, A. Lanzavecchia, and F. Sallusto, "Kinetics of dendritic cell activation: impact on priming of TH1, TH2 and nonpolarized T cells," Nature Immunology, vol. 1, no. 4, pp. 311-316, 2000.

[10] G. Trinchieri, “Interleukin-12: a proinflammatory cytokine with immunoregulatory functions that bridge innate resistance and antigen-specific adaptive immunity," Annual Review of Immunology, vol. 13, pp. 251-276, 1995.

[11] S. Miyazaki, F. Ishikawa, T. Fujikawa, S. Nagata, and K. Yamaguchi, "Intraperitoneal injection of lipopolysaccharide induces dynamic migration of Gr-1high polymorphonuclear neutrophils in the murine abdominal cavity," Clinical and Diagnostic Laboratory Immunology, vol. 11, no. 3, pp. 452-457, 2004.

[12] C. Morel, E. Badell, V. Abadie et al., "Mycobacterium bovis BCG-infected neutrophils and dendritic cells cooperate to induced specific T cell responses in humans and mice," European Journal of Immunology, vol. 38, no. 2, pp. 437-447, 2008.

[13] S. W. Seow, J. N. B. Rahmat, A. A. K. Mohamed, R. Mahendran, Y. K. Lee, and B. H. Bay, "Lactobacillus species is more cytotoxic to human bladder cancer cells than Mycobacterium Bovis (bacillus Calmette-Guerin)," The Journal of Urology, vol. 168, no. 5, pp. 2236-2239, 2002.

[14] I. Von Ossowski, J. Reunanen, R. Satokari et al., "Mucosal adhesion properties of the probiotic lactobacillus rhamnosus GG SpaCBA and SpaFED pilin subunits," Applied and Environmental Microbiology, vol. 76, no. 7, pp. 2049-2057, 2010.

[15] J. N. Rahmat, K. Esuvaranathan, and R. Mahendran, "Bacillus Calmette-Guérin induces cellular reactive oxygen species and lipid peroxidation in cancer cells," Urology, vol. 79, no. 6, pp. 1411.e15-1411.e20, 2012.

[16] M. Kandasamy, B.-H. Bay, Y.-K. Lee, and R. Mahendran, "Lactobacilli secreting a tumor antigen and IL15 activates neutrophils and dendritic cells and generates cytotoxic $\mathrm{T}$ lymphocytes against cancer cells," Cellular Immunology, vol. 271, no. 1, pp. 89-96, 2011.

[17] S. Hedlund, A. Persson, A. Vujic, K. F. Che, O. Stendahl, and M. Larsson, "Dendritic cell activation by sensing Mycobacterium tuberculosis-induced apoptotic neutrophils via DCSIGN," Human Immunology, vol. 71, no. 6, pp. 535-540, 2010.

[18] M. Lanzinger, B. Jürgens, U. Hainz et al., "Ambivalent effects of dendritic cells displaying prostaglandin E2-induced indoleamine 2,3-dioxygenase," European Journal of Immunology, vol. 42, no. 5, pp. 1117-1128, 2012.

[19] A. Shimabukuro-Vornhagen, T. M. Liebig, T. Koslowsky, S. Theurich, and M. S. von Bergwelt-Baildon, "The ratio between dendritic cells and $T$ cells determines whether prostaglandin $\mathrm{E}_{2}$ 
has a stimulatory or inhibitory effect," Cellular Immunology, vol. 281, no. 1, pp. 62-67, 2013.

[20] H. Harizi, M. Juzan, V. Pitard, J.-F. Moreau, and N. Gualde, "Cyclooxygenase-2-issued prostaglandin E2 enhances the production of endogenous IL-10, which down-regulates dendritic cell functions," Journal of Immunology, vol. 168, no. 5, pp. 22552263, 2002.

[21] N. Baba, S. Samson, R. L. Bourdet-Sicard, M. Rubio, and M. Sarfati, "Commensal bacteria trigger a full dendritic cell maturation program that promotes the expansion of non-Tr1 suppressor T cells," Journal of Leukocyte Biology, vol. 84, no. 2, pp. 468-476, 2008.

[22] T. Takahashi, A. Kushiro, K. Nomoto et al., "Antitumor effects of the intravesical instillation of heat killed cells of the lactobacillus casei strain shirota on the murine orthotopic bladder tumor MBT-2," Journal of Urology, vol. 166, no. 6, pp. 2506-2511, 2001.

[23] M. Mohamadzadeh, S. Olson, W. V. Kalina et al., "Lactobacilli activate human dendritic cells that skew $\mathrm{T}$ cells toward T helper 1 polarization," Proceedings of the National Academy of Sciences of the United States of America, vol. 102, no. 8, pp. 2880-2885, 2005.

[24] H. R. Christensen, H. Frøkiær, and J. J. Pestka, "Lactobacilli differentially modulate expression of cytokines and maturation surface markers in murine dendritic cells," The Journal of Immunology, vol. 168, no. 1, pp. 171-178, 2002.

[25] S. Cai, B. H. Bay, Y. K. Lee, J. Lu, and R. Mahendran, "Live and lyophilized Lactobacillus species elicit differential immunomodulatory effects on immune cells," FEMS Microbiology Letters, vol. 302, no. 2, pp. 189-196, 2010.

[26] H. L. Tytgat, N. H. van Teijlingen, R. M. Sullan et al., "Probiotic gut microbiota isolate interacts with dendritic cells via glycosylated heterotrimeric pili," PLoS One, vol. 11, no. 3, article e0151824, 2016.

[27] T. Shimosato, H. Kitazawa, S. Katoh et al., "Augmentation of $\mathrm{T}_{\mathrm{H}}-1$ type response by immunoactive AT oligonucleotide from lactic acid bacteria via Toll-like receptor 9 signaling," Biochemical and Biophysical Research Communications, vol. 326, no. 4, pp. 782-787, 2005.

[28] S. Ichikawa, M. Miyake, R. Fujii, and Y. Konishi, "MyD88 associated ROS generation is crucial for Lactobacillus induced IL-12 production in macrophage," PLoS One, vol. 7, no. 4, article e35880, 2012.

[29] K. Shida, J. Kiyoshima-Shibata, M. Nagaoka, K. Watanabe, and M. Nanno, "Induction of interleukin-12 by Lactobacillus strains having a rigid cell wall resistant to intracellular digestion," Journal of Dairy Science, vol. 89, no. 9, pp. 3306-3317, 2006.

[30] V. Abadie, E. Badell, P. Douillard et al., "Neutrophils rapidly migrate via lymphatics after Mycobacterium bovis BCG intradermal vaccination and shuttle live bacilli to the draining lymph nodes," Blood, vol. 106, no. 5, pp. 1843-1850, 2005.

[31] A. M. DeCathelineau and P. M. Henson, "The final step in programmed cell death: phagocytes carry apoptotic cells to the grave," Essays in Biochemistry, vol. 39, pp. 105-117, 2003.

[32] C. Alfaro, N. Suarez, C. Oñate et al., "Dendritic cells take up and present antigens from viable and apoptotic polymorphonuclear leukocytes," PLoS ONE, vol. 6, no. 12, Article ID e29300, 2011.

[33] L. M. Stuart, M. Lucas, C. Simpson, J. Lamb, J. Savill, and A. Lacy-Hulbert, "Inhibitory effects of apoptotic cell ingestion upon endotoxin-driven myeloid dendritic cell maturation," Journal of Immunology, vol. 168, no. 4, pp. 1627-1635, 2002.

[34] A. Camporeale, A. Boni, G. Iezzi et al., "Critical impact of the kinetics of dendritic cells activation on the in vivo induction of tumor-specific T lymphocytes," Cancer Research, vol. 63, no. 13, pp. 3688-3694, 2003.

[35] K. Steinbrink, M. Wölfl, H. Jonuleit, J. Knop, and A. H. Enk, "Induction of tolerance by IL-10-treated dendritic cells," The Journal of Immunology, vol. 159, no. 10, pp. 4772-4780, 1997.

[36] D. L. Commeren, P. L. Van Soest, K. Karimi, B. Löwenberg, J. J. Cornelissen, and E. Braakman, "Paradoxical effects of interleukin-10 on the maturation of murine myeloid dendritic cells," Immunology, vol. 110, no. 2, pp. 188-196, 2003.

[37] L. Faulkner, G. Buchan, and M. Baird, "Interleukin-10 does not affect phagocytosis of particulate antigen by bone marrowderived dendritic cells but does impair antigen presentation," Immunology, vol. 99, no. 4, pp. 523-531, 2000.

[38] W. L. W. Chang, N. Baumgarth, M. K. Eberhardt et al., "Exposure of myeloid dendritic cells to exogenous or endogenous IL10 during maturation determines their longevity," The Journal of Immunology, vol. 178, no. 12, pp. 7794-7804, 2007. 


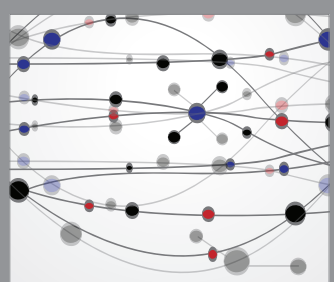

The Scientific World Journal
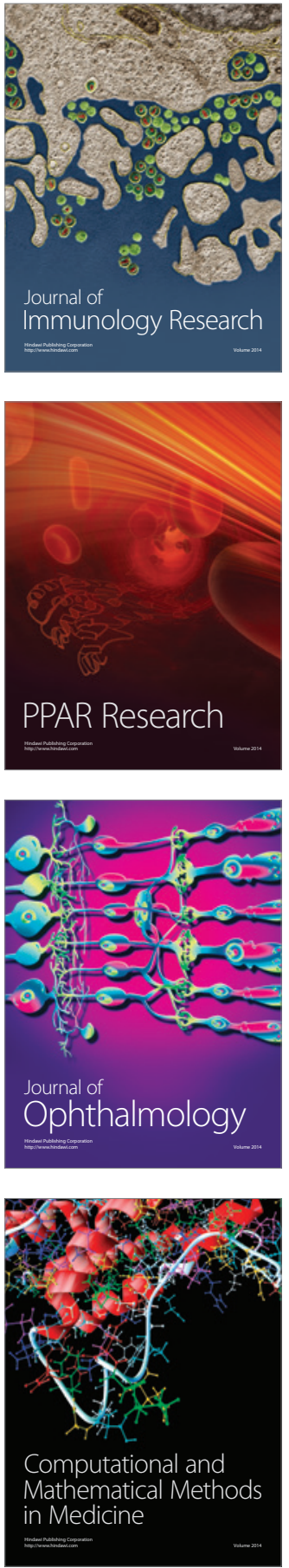

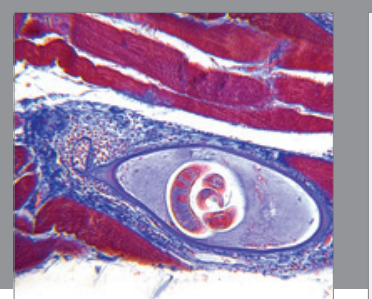

Gastroenterology Research and Practice

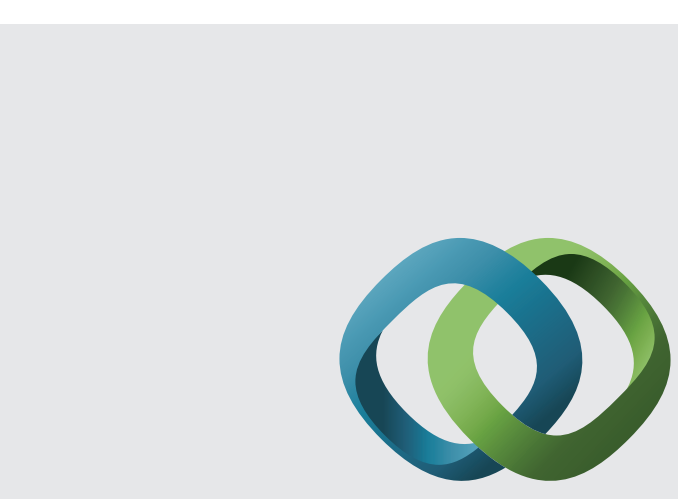

\section{Hindawi}

Submit your manuscripts at

http://www.hindawi.com
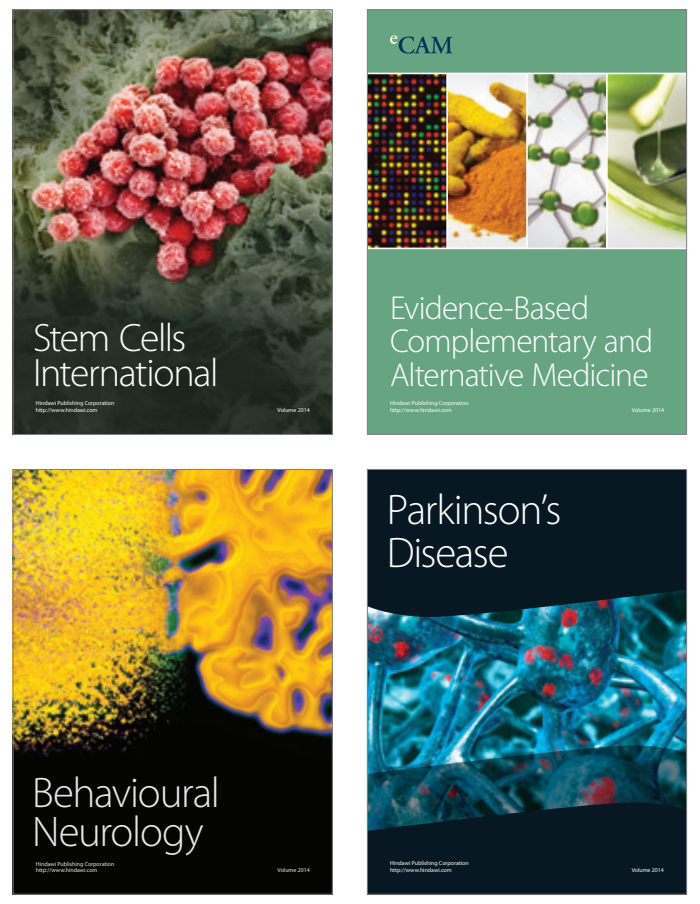
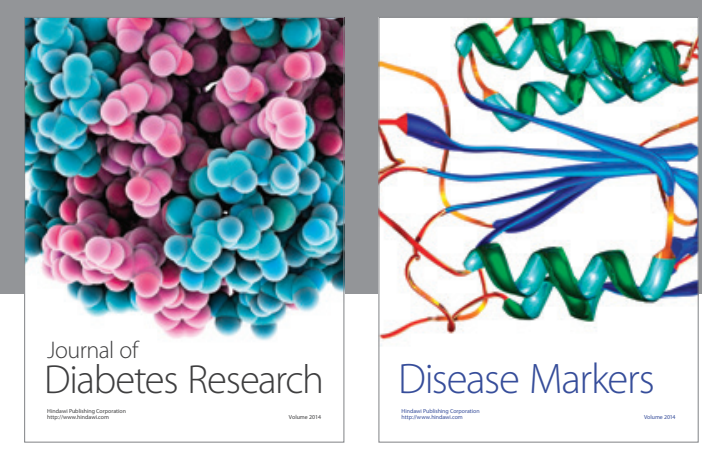

Disease Markers
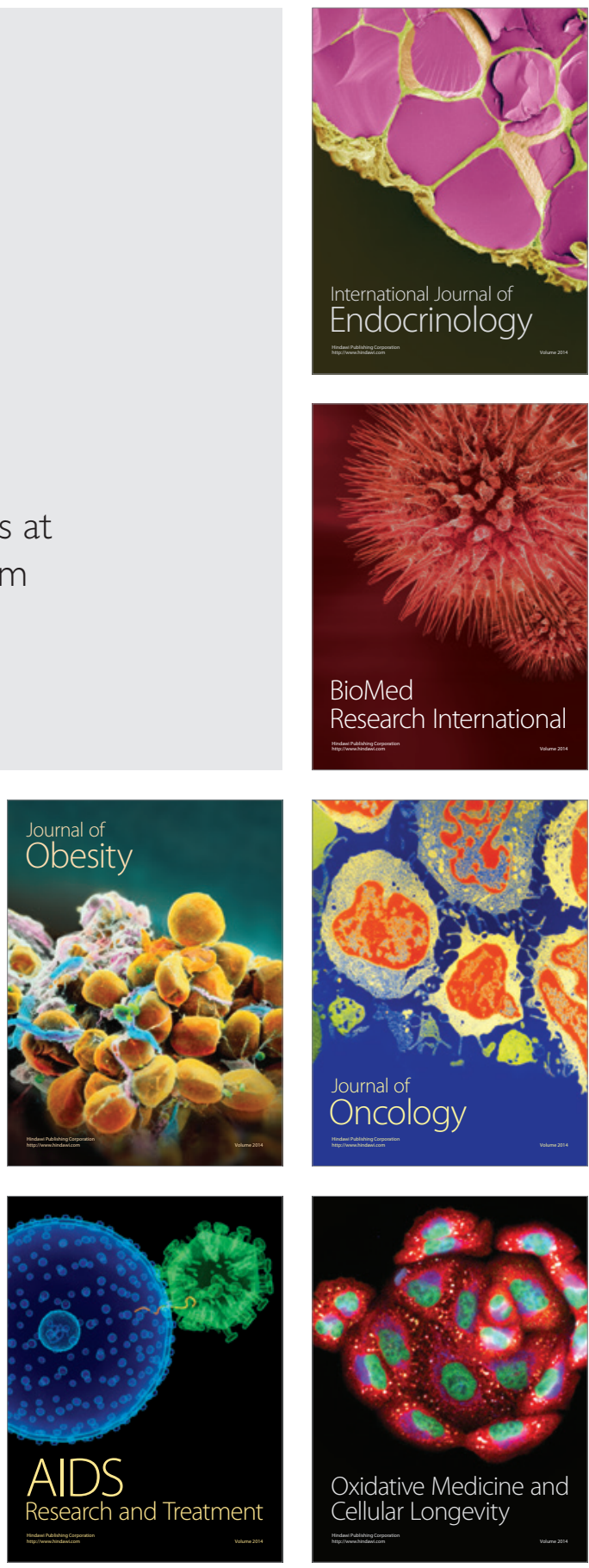